\title{
Transfusion-associated microchimerism in traumatic patients
}

\author{
Ying Cui ${ }^{\Delta}$, Rujuan Bao ${ }^{\Delta}$, Shijie Mu* \\ Department of Blood Transfusion, The Second Affiliated Hospital of Air Force Medical University, Xi'an, Shaanxi, 710038, China
}

\begin{abstract}
Transfusion-associated microchimerism (TA-MC) is the stable persistence of an allogeneic cell population, resulting from allogeneic blood transfusion (often resulting after trauma). Currently TA-MC is not completely understood, needing further study to reveal its underlying mechanism. This article reviews the immune tolerance mechanism of TA-MC; factors which lead to the appearance of TA-MC; clinical implications of microchimerism; and the latest diagnostic methods of TA-MC.
\end{abstract}

Keywords: transfusion-associated microchimerism, blood transfusion, trauma

\section{TRANSFUSION-ASSOCIATED MICROCHI- MERISM(TA-MC)}

The word "chimera" first appeared in Homer's Iliad, describing a fire-breathing beast. This beast had the head of lion, the body of goat and the tail of snake and was believed to be a sign of disaster ${ }^{[1]}$. In the field of science, "chimera" is a hybrid single group consisting of genetically different cells. "Microchimerism" is the presence of a small number of heterogeneous cells $(<5 \%)$ in a genetically different host individual. There are a variety of conditions that can cause microchimerism, including pregnancies where a mother retains a small number of fetus cells, and the fetus is born with a few cells genetically different from their mothers $^{[2,3]}$, solid organ and hematopoietic stem cell transplantation $^{[4]}$ and blood transfusion ${ }^{[5]}$. As its name implies, transfusion-associated microchimerism (TA$\mathrm{MC})$ is the persistent allogeneic exposure to foreign cells caused by blood transfusion, particularly follow-

\footnotetext{
*Correspondence to: Shijie Mu, Department of Blood Transfusion, The Second Affiliated Hospital of Air Force Medical University, Xi'an 710038, Shaanxi, China. Tel/Fax: +86-29-89661581. E-mail: musj1963@163.com.

$\Delta$ The authors contributed equally to this manuscript.

Conflict of interests: The authors declared no conflict of interests.
}

ing severe trauma ${ }^{[6]}$.

Research concerning TA-MC has gone through three landmark phases: (1) In the 1970s, Schechter et al. demonstrated karyotype evidence of donor leukocyte proliferation in recipients within 7 days (failing to increase over 7 days) after transfusion ${ }^{[7,8]}$. (2) Lee et al. conducted a case-control study which replicated early experience: persistent TA-MC appeared in transfused patients with trauma within 20 years ${ }^{[9]}$. (3) Blood transfusions in patients with severe traumatic injuries result in a high incidence $(20 \% \sim 40 \%)$ of TA-MC persistence over a long time ${ }^{[10]}$.

\section{TA-MCFOLLOWING TRAUMATIC INJURY}

Many studies observed that TA-MC is closely related to blood transfusion after traumatic injury. PCR HLA typing and mixed leukocyte reaction (MLR) were performed by Lee et al. finding that 7 out of 10 female trauma patients exhibited a multilineage persistence of male donor white blood cells from 6 months to 1.5 years $^{[9]}$. Utter et al. found post trauma transfusion is associated with evidence of microchimerism among over half of recipients (24 out of 45 subjects, $53 \%)^{[6]}$. Additionally, Utter et al. also found that TA-MC is a common phenomenon post transfusion after the occurrence of trauma ${ }^{[11]}$. In brief, the percentage of TA-MC in civilian trauma patients 
receiving allogenic red blood cells(RBCs) transfusions is about $20 \% \sim 40 \%$. Dunne et al. explored that the incidence of TA-MC in combat casualties receiving fresh whole blood ( 3 of $6,50 \%)$, platelets ( 4 of 8 , $50 \%$ ) and RBCs (3 of 8, 38\%), suggesting that TA$\mathrm{MC}$ in transfused soldiers is very common ${ }^{[10]}$.

\section{THE MECHANISM OF TA-MC}

The mechanism of TA-MC has not yet been fully revealed. However, changes in the immune system caused by trauma and blood transfusion may be the underlying cause ${ }^{[1]}$. Short-term immunosuppression and complications due to infections are major causes of death in post trauma patients ${ }^{[12]}$. Early studies have shown that trauma and stimulation impair innate and adaptive immunity function, involving T cells, B cells, and monocytes. Studies in vivo have shown that Th2 type cytokines such as IL-4 and IL-10 increase while mice down-regulate Thl type cytokines (IL-2, IFN- $\gamma$ ) and decrease $\mathrm{T}$ cell proliferative response when stimulated with phytohemagglutinin (PHA), pokeweed mitogen (PWM) or bacterial lipopolysaccharide (LPS) for 5 to 10 days after trauma ${ }^{[13-15]}$, which is consistent with findings in humans ${ }^{[16,17]}$. In addition, $\mathrm{IgG} /$ IgM reduced expression. Although the monocytes increased, they inhibited the secretion of IFN $-\gamma$ and expression of HLA-DR. Early studies also indicated that macrophage up-regulated the expression of prostaglandin $\mathrm{E} 2^{[18]}$, IL-6 $6^{[19]}$ and IL- $10^{[20]}$ following the inhibition of T cells; Toll-like receptors (TLR)-2 and TLR-4 induced the augment of IL-1, IL-6 and $\mathrm{TNF}-\alpha$ secretion by macrophages in the dysregulation of innate immune response ${ }^{[21]}$; and IL-10 showed an elevated expression caused by macrophages after severe trauma ${ }^{[22]}$. At the molecular level, peripheral blood mononuclear cells secrete less NF- $\mathrm{KB}$, which impairs the activation of $\mathrm{T}$ cells ${ }^{[23]}$. All of the above studies focused on the inhibition of Th2 cytokines to $\mathrm{T}$ cells' immune responses following trauma. Therefore, post-trauma immune system disorder may explain why TA-MC happens to trauma patients after receiving blood transfusions. Traumatic injury and blood transfusion are critical immune stimuli which induce immune regulation to form a micro-environment for the development of TA-MC. In a case-control study, TA-MC patients who underwent post trauma transfusion had a weak lymphocyte response compared to patients with trauma, but had not undergone blood transfusion. The above studies are limited to the analysis of "short-term TA-MC". However, how these chimeras keep stable in the host's immune system for decades is still unclear. One explanation is that the host's immune tolerance may induce body refractory to the chimeras ${ }^{[9]}$.

\section{THE FACTORS WHICH LEAD TO THE AP- PEARANCE OF TA-MC}

To date, almost all studies have shown that TAMC only occurs in patients who have been transfused after traumatic injury. However, not all post-traumatic blood transfusions cause TA-MC, so further research is needed to find other related factors, such as: HLA compatibility, severity of trauma, variability of transfusion products, etc. Ultimately, the development of TA-MC depends on how these factors influence immune responses ${ }^{[1]}$. In recent years, research progress concerning the factors affecting the formation of TA$\mathrm{MC}$ is exemplified by the following examples:

(1) In a prospective cohort study, Bloch et al. set out to determine if transfusion in the peripartum period results in lasting high-level TA-MC. Evidence of transient TA-MC was found in 2 of 22 women who received blood transfusion within 48 hours. However, no persistent TA-MC was detected at 6 weeks or 6 months post transfusion ${ }^{[24]}$.

(2) A population-based case-control study conducted by Müller et al. found that microchimerism in young expectant mothers may originate from transfusion during discontinued pregnancy ${ }^{[25]}$.

(3) A study conducted by Sanchez et al. aimed to evaluate whether 409 adult and pediatric females receiving leuko-reduced and mostly irradiated allogeneic RBC and platelet transfusions would develop TA-MC. They found that 40 of 207 (19\%) of adult and 44 of $202(22 \%)$ pediatric female blood recipients went on to develop low level non-persistent MC, demonstrating that leuko-reduced and mostly irradiated allogeneic RBC and platelet transfusions are able to prevent the adult and pediatric recipient from developing enduring TA-MC ${ }^{[26]}$. Conversely, from a study of Australian trauma patients $(n=86)$ who received RBC transfusions from 2000 to 2012: 9 of 55 $(16.3 \%)$ patients transfused with non-leukodepleted blood components and 3 of 31 (9.6\%) transfused with leukodepleted RBC units developed persistent TAMC. Although non-leukodepleted blood components are now commonly used in Australia, clinical results show that the incidence of TA-MC has not changed significantly ${ }^{[27]}$. Notably, half of the patients included in the above study were recorded as splenic injury or required splenectomy during transfusion. From this, we believe the spleen may play a pivotal role in filtering abnormal cells and producing antibodies and lymphocytes. In a suggested splenic injury scenario, the donor cells may still be able to escape the host's immune system to form TA-MC, even if the recipient 
has received non-leukodepleted blood components.

\section{CLINICAL IMPLICATON OF TA-MC}

The negative clinical significance of "microchimerism" is thought to be related to autoimmune diseases ${ }^{[28]}$. Studies in support of this have found that fetal chimeric cells are commonly found in systemic lupus erythematosus (SLE) females with traumas. Significant differences were found between SLE traumatic patients and healthy controls $(P<0.001)$, while no statistically significant differences were found between traumatic SLE female patients and nontraumatic SLE female patients $(P=0.036)^{[29]}$. Therefore, the relationship between autoimmune diseases and micro-chimeras appears to be of the "chicken and egg" variety, where it seems impossible to determine a clear relationship ${ }^{[30]}$. Micro-chimerism may have some positive aspects: (1) "Repair hypothesis" suggests chimera cells are capable of differentiating into parenchymal cells in response to tissue injury, aiding ability for multi-lineage differentiation ${ }^{[29]}$. (2) Prenatal genetic diagnosis, which currently relies on invasive sampling e.g. chorionic villus biopsy and amniocentesis, both of which increase the risk of abortion. If fetal chimeric cells or cell-free DNA in the peripheral circulation of pregnant women can be accurately detected, pregnant women may be able to avoid highrisk sampling ${ }^{[31,32]}$.

\section{DIAGNOSTIC ANALYSIS METHOD FOR TA-MC}

Early technologies for detecting TA-MC rely mainly on: (1) PCR targeting the Y-chromosome, which is a sex-based method. (2) Quantitative realtime PCR, which has greatly improved the detection sensitivity and specificity of TA-MC ${ }^{[33]}$. (3) HLA-DR alleles assay, which recognizes insertion/deletion (InDel) polymorphisms ${ }^{[34]}$. The above methods' detailed instructions have been previously published. This review, however, describes a method for sorting microchimerism in blood cells by flow cytometry, based on human HLA antigen. The method outlined below was developed by Drabbels ${ }^{[35]}$ and Eikmans et al. ${ }^{[36]}$, who established the most efficient separation method, with a sorting ratio as low as $0.01 \%$. Here two different human monoclonal HLA antibodies were used in the assay, one for the chimeric cells, the other targeted background cells. After successful isolation, DNA analysis was used to detect the specificity of the HLA allele and Y-chromosome-RT-qPCR was performed to verify the purity of the isolated cell population. When compared with pre-treated samples, chimeric DNA markers' PCR signals were significantly enhanced on positive population. Due to these proper- ties, this method was successfully applied to isolate maternal micro-chimera cells from umbilical cord mononuclear cells. This new technology is able to maximize the enrichment of micro chimeric cells that may exist in the host, opening a new horizon for the study of micro chimeric cell's phenotype and function.

In summary, TA-MC research is still in its infancy, and scientists are still required to conduct further research to explore both its negative, positive effects and deeper mechanism, in order that it may be regulated or manipulated to the benefit of the patient.

\section{References}

[1] Bloch EM, Jackman RP, Lee TH, et al. Transfusion-associated microchimerism:the hybrid within. Transfus Med Rev, 2013, 27(1):10-20.

[2] Bianchi DW, Zickwolf GK, Weil GJ, et al. Male fetal progenitor cells persist in maternal blood for as long as 27 years postpartum. Proc Natl Acad Sci the U S A, 1996, 93(2):705-8.

[3] Cawthorne T, Foxwell PB. An antihistamine in the fenestration operation for otosclerosis. Br Med J, 1953, 2(4827):80.

[4] Adams KM, Nelson JL. Microchimerism: An investigative frontier in autoimmunity and transplantation. JAMA, 2004, 291(9):1127-31.

[5] Lee TH, Paglieroni T, Utter GH, et al. High-level longterm white blood cell microchimerism after transfusion of leukoreduced blood components to patients resuscitated after severe traumatic injury. Transfusion, 2010, 45(8):1280-90.

[6] Utter GH, Owings JT, Lee TH, et al. Blood transfusion is associated with donor leukocyte microchimerism in trauma patients. J Trauma, 2004, 57(4):707-8.

[7] Schechter GP, Whang-Peng J, Mcfarland W. Circulation of donor lymphocytes after blood transfusion in man. Blood, 1977, 49(4):651-6.

[8] Adams PT, Davenport RD, Reardon DA, et al. Detection of circulating donor white blood cells in patients receiving multiple transfusions. Blood, 1992, 80(2):551-5.

[9] Lee TH, Paglieroni T, Ohto H, et al. Survival of donor leukocyte subpopulations in immunocompetent transfusion recipients: frequent long-term microchimerism in severe trauma patients. Blood, 1999, 93(9):3127-39.

[10] Dunne JR, Lee TH, Burns C, et al. Transfusion-associated microchimerism in combat casualties. J Trauma, 2008, 64(2 Suppl): 92-7.

[11] Utter GH, Nathens AB, Lee TH, et al. Leukoreduction of blood transfusions does not diminish transfusion-associated microchimerism in trauma patients. Transfusion, 2006, 46(11):1863-9.

[12] O`Mahony JB, Palder SB, Wood JJ, et al. Depression of cellular immunity after multiple trauma in the absence of sepsis. J Trauma, 1984, 24(10):869.

[13] Faist E, Mewes A, Baker CC, et al. Prostaglandin E2 (PGE2)-dependent suppression of interleukin alpha (IL- 
2) production in patients with major trauma. J Trauma, 1987, 27(8):837-48.

[14] Quattrocchi KB, Frank EH, Miller CH, et al. Impairment of helper $\mathrm{T}$-cell function and lymphokine-activated killer cytotoxicity following severe head injury. $\mathrm{J} \mathrm{Neu-}$ rosurg, 1991, 75(5):766-73.

[15] Lyons A, Goebel A, Mannick JA, et al. Protective effects of early interleukin 10 antagonism on injury-induced immune dysfunction. Arch Surg, 1999, 134(12):1317-23.

[16] Puyana JC, Pellegrini JD, De AK, et al. Both T-helper-1and T-helper-2-type lymphokines are depressed in posttrauma anergy. J Trauma, 1998, 44(6):1037-45.

[17] Lyons A, Kelly JL, Rodrick ML, et al. Major injury induces increased production of interleukin-10 by cells of the immune system with a negative impact on resistance to infection. Ann Surg, 1997, 226(4):458-60.

[18] Miller-Graziano CL, Szabo G, Kodys K, et al. Aberrations in post-trauma monocyte (MO) subpopulation: role in septic shock syndrome. J Trauma, 1990, 30(12 Suppl):86-96.

[19] Durbin EA, Gregory MS, Messingham KAN, et al. The role of interleukin 6 in interferon- $\gamma$ production in thermally injured mice. Cytokine, 2000, 12(11):1669-75.

[20] Göebel A, Kavanagh E, Lyons A, et al. Injury induces deficient interleukin-12 production, but interleukin-12 therapy after injury restores resistance to infection. Ann Surg, 2000, 231(2):253-61.

[21] Paterson HM, Murphy TJ, Purcell EJ, et al. Injury primes the innate immune system for enhanced Toll-like receptor reactivity. J Immunol, 2003, 171(3):1473.

[22] Köller M, Clasbrummel B, Kollig E, et al. Major injury induces increased production of interleukin-10 in human granulocyte fractions. Langenbecks Arch Surg, 1998, 383(6):460-5.

[23] Adib-Conquy M, Asehnoune K, Moine P, et al. Longterm-impaired expression of nuclear factor $-\kappa \mathrm{B}$ and $\mathrm{I} \kappa \mathrm{B} \alpha$ in peripheral blood mononuclear cells of trauma patients. J Leukoc Biol, 2001, 70(1):30-8.

[24] Bloch EM, Busch MP, Lee TH, et al. Microchimerism in the transfused obstetric population. Vox Sang, 2015, 107(4):428-30.

[25] Müller AC, Jakobsen MA, Barington T, et al. Microchi- merism of male origin in acohort of Danish girls. Chimerism, 2016, 6(4):65-71.

[26] Sanchez R, Lee TH, Wen L, et al. Absence of transfusion-associated microchimerism in pediatric and adult recipients of leukoreduced and gamma-irradiated blood components. Transfusion, 2012, 52(5):936-45.

[27] Hirani R, Balogh ZJ, Lott NJ, et al. Leukodepleted blood components do not remove the potential for long-term transfusion-associated microchimerism in Australian major trauma patients. Chimerism, 2014, 5(3-4):86-93.

[28] Nelson JL. Microchimerism in human health and disease. Autoimmunity, 2003, 36(1):5-9.

[29] Kremer Hovinga IC, Koopmans M, Baelde HJ, et al. Tissue chimerism in systemic lupus erythematosus is related to injury. Anna Rheum Dis, 2007, 66(12):1568-73.

[30] Kremer Hovinga IC, Koopmans M, De HE, et al. Chimerism in systemic lupus erythematosus--three hypotheses. Rheumatology (Oxford), 2007, 46(2):200-8.

[31] Bianchi DW.Fetomaternal cell trafficking: a story that begins with prenatal diagnosis and may end with stem cell therapy. J Pediatr Surg, 2007, 42(1):12-8.

[32] Bianchi DW, Simpson JL, Jackson LG, et al. Fetal gender and aneuploidy detection using fetal cells in maternal blood: analysis of NIFTY I data. Prenat Diagn, 2002, 22(7):609-15.

[33] Ariga H, Ohto H, Busch MP, et al. Kinetics of fetal cellular and cell-free DNA in the maternal circulation during and after pregnancy: implications for noninvasive prenatal diagnosis. Transfusion, 2010, 41(12):1524-30.

[34] Utter GH, Owings JT, Lee TH, et al. Microchimerism in transfused trauma patients is associated with diminished donor-specific lymphocyte response. J Trauma, 2005, 58(5):925-31.

[35] Drabbels JJ, Van d KC, Kemps BM, et al. HLA-targeted flow cytometric sorting of blood cells allows separation of pure and viable microchimeric cell populations. Blood, 2011, 118(4):e149-55.

[36] Eikmans M, Claas FH. HLA-targeted cell sorting of microchimeric cells opens the way to phenotypical and functional characterization. Chimerism, 2011, 2(4):114-6.

(Received 11 April 2018, Revised 28 August 2018, Accepted 30 August 2018) 